\title{
A compliance questionnaire could discriminate among patients for drug taking behaviour and correct dosing in rheumatic diseases
}

de Klerk E, van der Heijde D, Landewé R, et al. The compliance-questionnaire-rheumatology compared with electronic medication event monitoring: a validation study. J Rheumatol 2003;30:2469-75

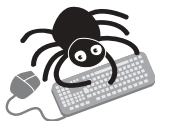

This article

contains extra text on the EBM website

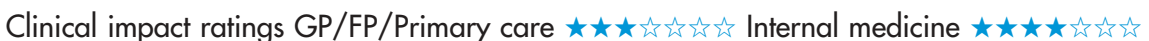

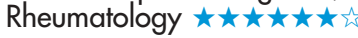

In patients with rheumatic diseases, how well does the compliance questionnaire rheumatology (CQR) predict compliance with taking medications and correct dosing compared with an electronic medication event monitoring system (MEMS)?

有 administered questionnaire.

Setting: outpatient rheumatology wards of 3 hospitals in the Netherlands.

Patients: 127 patients (mean age 61 y, 57\% women) who had rheumatoid arthritis and were taking sulphasalazine,

methotrexate, diclofenac, or naproxen; polymyalgia rheumatica and were taking prednisone or prednisolone; or gout and were taking colchicine, allopurinol, or benzbromaron. The drugs had to be first time prescriptions and taken as directed, not on demand. Patients were excluded if a caregiver was involved in the administration of medication.

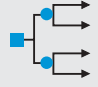

Prediction guide: patients completed the CQR in the first week after starting medication. The CQR contained 19 questions on drug taking behaviour (score range 0 [complete non-compliance] to 100 [perfect compliance]). Patients received a MEMS pill bottle which was filled by their pharmacist. The MEMS cap contained electronic circuitry that recorded the time and date of each opening and closing of the pill bottle. At 6 months (12 mo for gout), patients returned the MEMS pill bottle.

Outcomes: drug taking compliance (percentage of prescribed doses taken), and correct dosing (percentage of days on which the correct number of doses was taken). Satisfactory compliance was defined as drug taking compliance or correct dosing $>80 \%$.

Discriminant analysis of the compliance questionnaire rheumatology in detecting poor drug taking compliance and dosing in rheumatic diseases*

\begin{tabular}{|c|c|c|c|}
\hline Outcomes & $\begin{array}{l}\text { Sensitivity } \\
(95 \% \mathrm{CI})\end{array}$ & Specificity (Cl) & $+\mathrm{LR}(\mathrm{CI})$ \\
\hline $\begin{array}{l}\text { Drug taking } \\
\text { compliance } \leqslant 80 \%\end{array}$ & $62 \%(57$ to 67$)$ & $95 \%$ (92 to 97$)$ & 11.6 (6.7 to 20$)$ \\
\hline $\begin{array}{l}\text { Drug taking } \\
\text { compliance } \leqslant 50 \%\end{array}$ & $63 \%(57$ to 68$)$ & $97 \%$ (96 to 99 ) & 24.1 (7.9 to 73$)$ \\
\hline $\begin{array}{l}\text { Correct dosing } \\
\leqslant 80 \%\end{array}$ & $89 \%$ (85 to 92 ) & $70 \%(65$ to 75$)$ & $2.9(1.5$ to 5.9$)$ \\
\hline $\begin{array}{l}\text { Correct dosing } \\
\leqslant 50 \%\end{array}$ & $62 \%(57$ to 67$)$ & $94 \%(91$ to 96$)$ & $9.9(7.0$ to 14$)$ \\
\hline
\end{tabular}

For correspondence: Dr D van der Heijde, University Hospital Maastricht, Maastricht, The Netherlands. dhe@sint.azm.nl

Source of funding: Dutch Arthritis Association.

\section{MAIN RESULTS}

The mean CQR score was 76.6. Total CQR score did not correlate with drug taking compliance or correct dosing. However, a multiple regression analysis weighting scores assigned to individual items showed CQR items predicted drug taking compliance and correct dosing. The discriminant analyses of the CQR for detecting drug taking compliance and correct dosing $\leqslant 80 \%$ and $\leqslant 50 \%$ are in the table.

\section{CONCLUSION}

In patients with rheumatic diseases, the compliance questionnaire rheumatology detected poor drug taking compliance and dosing.

Commentary

A sking patients directly but non-judgmentally about their medication taking is the most useful way to assess compliance in clinical practice. ${ }^{1}$ Over the past 30 years, studies using different questionnaires and different methods of validation have consistently shown that the sensitivity of patient self reports of non-compliance is low, but the positive predictive value of such reports is high. In other words, patient reports of non-compliance are generally accurate, but many patients who are not compliant will not say so. The findings of de Klerk et al confirm this observation.

The use of electronic medication monitoring, the most accurate single measure of compliance, is a strength of their study, but the 12 minutes required to complete the CQR and the need for weighting of responses to enhance its predictive value are barriers to its clinical use. The finding that $33 \%$ of those offered the CQR did not provide analysable responses raises a concern about potential bias, since people non-compliant with surveys about their compliance may also be non-compliant with their pills. Additionally, the data were collected within 2 weeks of initiating a new rheumatological drug, so that these patient responses may be most predictive of their early decisions about whether to continue the drugs rather than their long term persistence with treatment.

Clinicians and researchers should remember the adage that "accurate measurements of compliance are not easy; easy measurement of compliance is not accurate. ${ }^{\prime 2}$ In the search for a clinically useful yet accurate compliance measure, de Klerk et al have validated a survey that identifies a subset of patients who do not take their medications as prescribed. Their finding that 4 of the 19 questions in the survey explain most of the variance in compliance raises the hope that a "short form" of their measure might be more readily incorporated into rheumatology practice (see web extra).

John F Steiner, MD, MPH University of Colorado Denver, Colorado, USA

1 Stephenson BJ, Rowe BH, Haynes RB, et al. The rational clinical examination. Is this patient taking the treatment as prescribed? JAMA 1993:269:2779-81.

2 Haynes RB. Patient compliance then and now [editorial]. Patient Educ Couns $1987 ; 10: 103-5$. 